\title{
Impressive response and long- term survival in a patient with metastatic extraskeletal myxoid chondrosarcoma treated with radiotherapy and trabectedin: a synergistic activity
}

The extraskeletal myxoid chondrosarcoma (EMC) is a rare malignant accounting for less than $3 \%$ of soft tissue tumors. EMC is considered an intermediate aggressive neoplasm with a slow-growing and indolent clinical course. Nevertheless, local recurrence is frequent and distant metastases arise in about $50 \%-90 \%$ of cases. For these patients therapeutic options are scarce due to the poorly sensitivity of EMC to cytotoxic chemotherapy and targeted therapy.

We report the case of a caucasian adult man with bulky locoregional and mediastinal disease resistant to antracyclinbased chemotherapy and targeted therapy. Then, a third line chemotherapy with trabectedin was used obtaining temporary disease stabilization. Due to arising of symptoms and modest increase of lesions, radiotherapy was added with four consecutive treatments on right gluteal mass, large right and left paravertebral lesions, and mediastinal bulky disease, respectively. Rapidly, near complete regression of masses was noted. After 41 months from metastasis and 28 after beginning of chemotherapy with trabectedin and complementary radiotherapy, the patient is in good clinical conditions and the disease under control.

In our experience, radiotherapy is a valid option in metastatic ECM and when associated with trabectedin a potent synergistic effect could be obtained.

Keywords: Extraskeletal myxoid chondrosarcoma, radiotherapy, trabectedin, long-term survival

\section{Introduction}

The extraskeletal myxoid chondrosarcoma (EMC) is a rare malignant mesenchimal neoplasm accounting for less than $3 \%$ of soft tissue tumors [1].

It occurs commonly in middle aged adults and is usually developed in the deep parts of proximal extremities and limb girdles with occurrence ratio between male and female of $2: 1$ with a peak occurrence in the fifth and sixth decades $[2,3]$.

It is classified as a tumor of uncertain differentiation and is described as a distinct clinical, histological, immunohistochemical, genetical and evolutive entity. Despite its name, EMC does not exhibit any cartilaginous differentiation. Microscopically, EMC can be subdivided into a conventional welldifferentiated and a cellular high-grade EMC, the latter being marked by the presence of a predominantly epithelioid morphology, high mitotic rate and necrosis [4]. Cases of dedifferentiated ECM have also been reported [5].
The diagnostic key is morphologically supported by immunohistochemistry and genetics $[9,22]$ that allow differentiating it from other tumours with myxoid stroma and from chordoma [4].

Clinically, EMC is considered an intermediate aggressive neoplasm with a slowgrowing and indolent clinical course. In fact, survival of patients with EMC is longer than other soft tissue sarcoma patients with a median overall survival of about 3 years and with many patients being very long survivors. Nevertheless, local recurrence is frequent and distant metastases arise in about $50 \%-90 \%$ of cases, usually in lungs, soft tissue, lymph nodes, bone, and brain [3, 6-7]. The mean survival after onset of metastases is about 3 years $[3,8]$.

Available literature reports EMC as a disease poorly sensitive to cytotoxic chemotherapy and targeted therapy [9-12]. Also radiotherapy has a controversial role in EMC both in adjuvant and metastatic sitting.
Ruggero Filannino ${ }^{1}$, Annalisa Nardone ${ }^{2}$, Livia Fucci ${ }^{3}$, Andrea Armenio ${ }^{4}$, Federica De Luca ${ }^{5}$, Francesco Figliuolo 4 , Francesco Macina ${ }^{6}$, Eustachio Ruggieri ${ }^{7}$, Stefania Tommasi ${ }^{8}$, Michele Traversa ${ }^{5}$, Umberto Orsini ${ }^{9}$, Anna Albano ${ }^{1}$, Roberta Carbonara ${ }^{10}$ and Michele Guida ${ }^{1 *}$

${ }^{1}$ Unit of Medical Oncology, University of Bari Aldo Moro, Bari, Italy

${ }^{2}$ Unit of Radiotherapy, University of Bari Aldo Moro, Bari, Italy

${ }^{3}$ Unit of Anatomy Patholoy, University of Bari Aldo Moro, Bari, Italy

${ }^{4}$ Unit of Plastic Surgery, University of Bari Aldo Moro, Bari, Italy 
We describe a metastatic extraskeletal myxoid chondrosarcoma patient with a high locoregional extension and bulky mediastinal adenophaties resistant to conventional chemotherapy and targeted therapy that responded impressively to radiotherapy in association with trabectedin.

\section{Case Report}

We report the case of a 65-year-old trader who has been suffering from hypothyroidism for about 5 years in hormone replacement therapy.

In September 2006 he underwent incisional biopsy for the appearance of swelling in the left thigh and the diagnosis of extra skeletal myxoid chondrosarcoma was formulated (FIGURE 1). Then an extensive resection of the lesion was performed with subsequent complementary radiotherapy (total dose 50 Gray). In 2012 there was evidence of millimetric multiple bilateral lesions suspected for metastases that were evaluated stable in numerous subsequent TACTB controls. Due to good clinical condition, patient interrupted follow up for some years but in November 2014 he noted the appearance of new lesions on the back and right gluteal region. A new TAC-TB showed rough swelling of soft tissue in the right gluteal region of $16 \times 11 \mathrm{~cm}$ and right dorsal paravertebral region of the size of $6 \times 15 \mathrm{~cm}$, and multiple conglomerated mediastinal adenopathies of overall dimensions of about $7 \times 12 \times 17 \mathrm{~cm}$. For this reason, the patient underwent incisional biopsy of gluteal mass and trans-bronchial biopsy of mediastinal lymph nodes with confirmation of the diagnosis of myxoid chondrosarcoma. In February 2015 he therefore started systemic therapy within a clinical trial with pazopanib $800 \mathrm{mg} /$ day administered orally. After 7 months of such therapy, treatment was stopped due to disease progression to all sites of disease. He then began chemotherapy with adriamycin $25 \mathrm{mg} / \mathrm{mq}$ day $1,8,15$ every 28 days, however the revaluation with TAC-TB performed after 5 cycles of therapy showed new disease progression (mediastinal adenopathic conglomerate $7 \times 12 \times 17 \mathrm{~cm}$, lesion

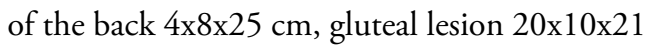
$\mathrm{cm})$. Therefore, in March 2016, the patient began a third-line systemic therapy with trabectedine $1.2 \mathrm{mg} / \mathrm{mq}$ in continuous $24-$ hour infusion every 28 days instead of every 21 days, considering previous multiple therapies lines already used. For palliative purposes due to appearance of pain and encumbrance on the right gluteal lesion, we decided to treat the patient with radiotherapy. A total dose of $39 \mathrm{~Gy}$ in 13 fractions of 3 Gy was delivered with four oblique X-Ray beams (FIGURE 2) with a rapid and impressive reduction of its size. At the end of radiation therapy we reported the absence of acute toxicity. The first revaluation TAC-TB performed in June 2016 showed a significant reduction in gluteal localization $(12 \times 5 \times 10 \mathrm{~cm}$ vs. $20 \times 10 \times 21 \mathrm{~cm})$ and substantial stationarity of the other lesions. The patient continued chemotherapy with trabectidine at the same doses and timing. A new TAC-TB in February
${ }^{5}$ Unit of Radiology, University of Bari Aldo Moro, Bari, Italy

${ }^{6}$ Unit of Interventional Radiology, University of Bari Aldo Moro, Bari, Italy

'Unit of Surgery, University of Bari Aldo Moro, Bari, Italy

${ }^{8}$ Unit of Molecular Diagnostics and Pharmacogenetics; University of Bari Aldo Moro, Bari, Italy

${ }^{9}$ Unit of Orthopedy - I.R.C.C.S "Giovanni Paolo II", Bari, Italy

${ }^{10}$ Unit of Radiotherapy University of Bari Aldo Moro, Bari, Italy

*Author for correspondence: micguida@libero.it
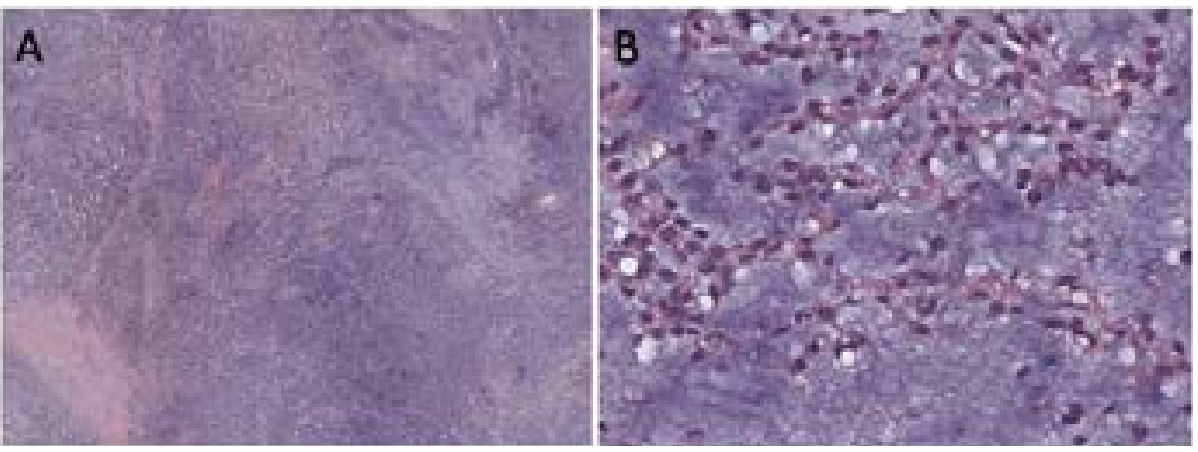

FIGURE 1: Histophatological features.

A. Tumor has a multinodular architecture with fibrous septa and abundant pale blue myxoid or chondromyxoid matrix rich in solfate proteoglycans (Hematoxylin and eosin staining, magnification $=10 x$ ).

B. Cells characteristically form cords with a modest amount of deeply eosinophilic o vacuolated cytoplasm and uniform round to oval nuclei. No mitoses were identified (Hematoxylin and eosin staining, magnification $=40 x$ ). 
2017 showed a further reduction of the gluteal lesion that resulted of $2 \times 1.5 \mathrm{~cm}$ (FIGURE 3) and a still stationarity of mediastinal-pulmonary disease and soft tissue lesion on the back. In consideration of the excellent result on the gluteal lesion, after 15 cycles of trabectedin and in the presence of stable disease, we also proposed a new radiation treatment on the right paravertebral dorsal lesion that was performed in May 2017 with total dose of 32 Gy in 8 fractions of $4 \mathrm{~Gy}$. The treatment plan for this lesion (mean volume $409.7 \mathrm{cc}$ ) was with two oblique X-Ray beams (FIGURE 4). Chemotherapy with trabectedin was continued. A new TAC-TB revaluation in August 2017 showed the onset of a new left cervical paravertebral lesion of $1.7 \times 3.5$ $\mathrm{cm}$ and substantial stability of the other lesions. In November 2017, in consideration of the increase in size of the left paravertebral lesion, patient performed a new cycle of radiotherapy. A total dose of 32 Gy in 8 fractions of 4 Gy was delivered with a single electron-beam $(20 \mathrm{MeV})$ (FIGURE 5). The last TAC-TB re-evaluation of March 2018 showed stability of multiple bilateral lung nodules of maximum size of $2 \mathrm{~cm}$, however a slight increase in mediastinal lymph node size extending $13 \times 9 \times 21 \mathrm{~cm}$ was described with the appearance of a progressive dyspnea requiring continuous oxygen-therapy. For these reasons the patient underwent new radiotherapic treatment on mediastinum (total dose of $30 \mathrm{~Gy}$ in 10 fractions). To ensure an adequate dosesparing of previously irradiated areas, a Rapid Arc plan with two arches and daily imageguided radiotherapy were performed (FIGURE 6) with a progressive amelioration of dyspnea.

Up to present, the patient has performed
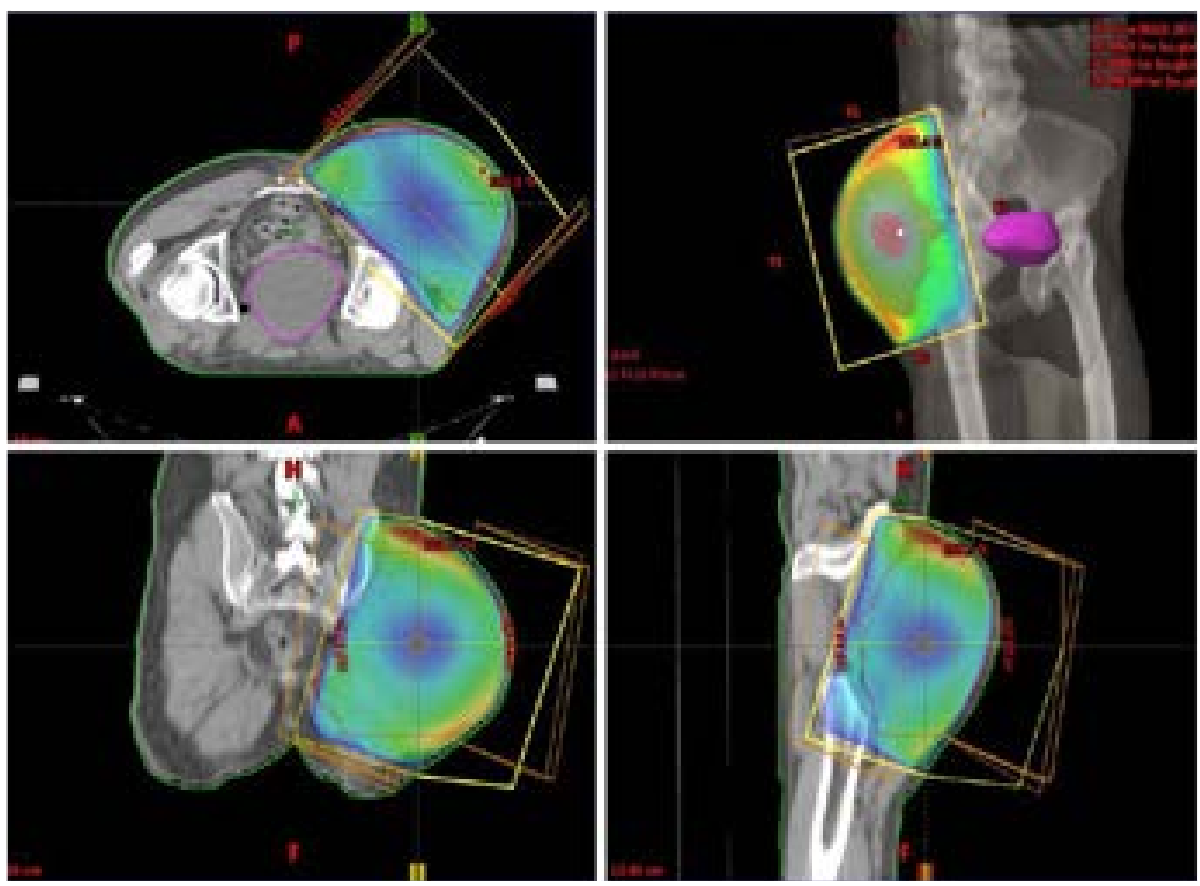

FIGURE 2: Treatment plan for right gluteal lesion.
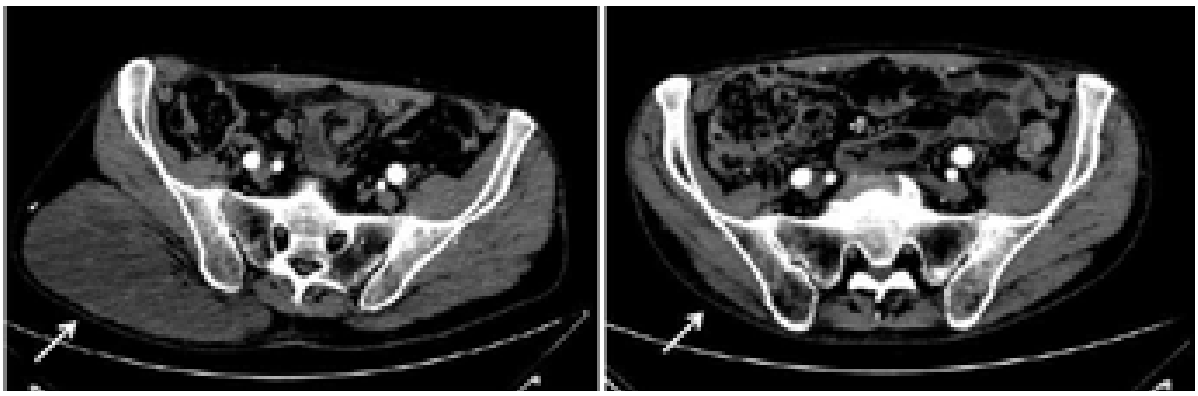

FIGURE 3: Impressive reduction of the gluteal lesion after radiotherapy (see arrows). 

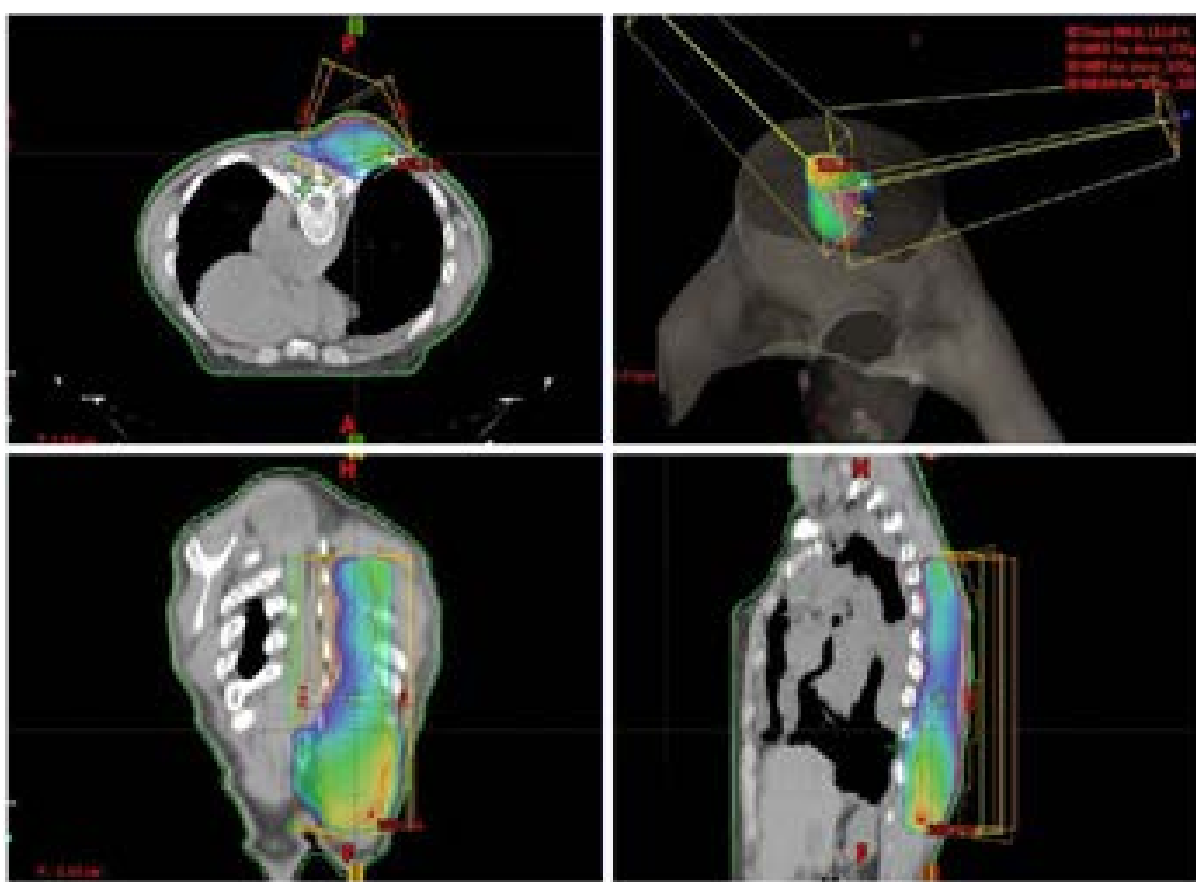

FIGURE 4: Treatment plan for right paravertebral dorsal lesion with dose-distribution in color wash.
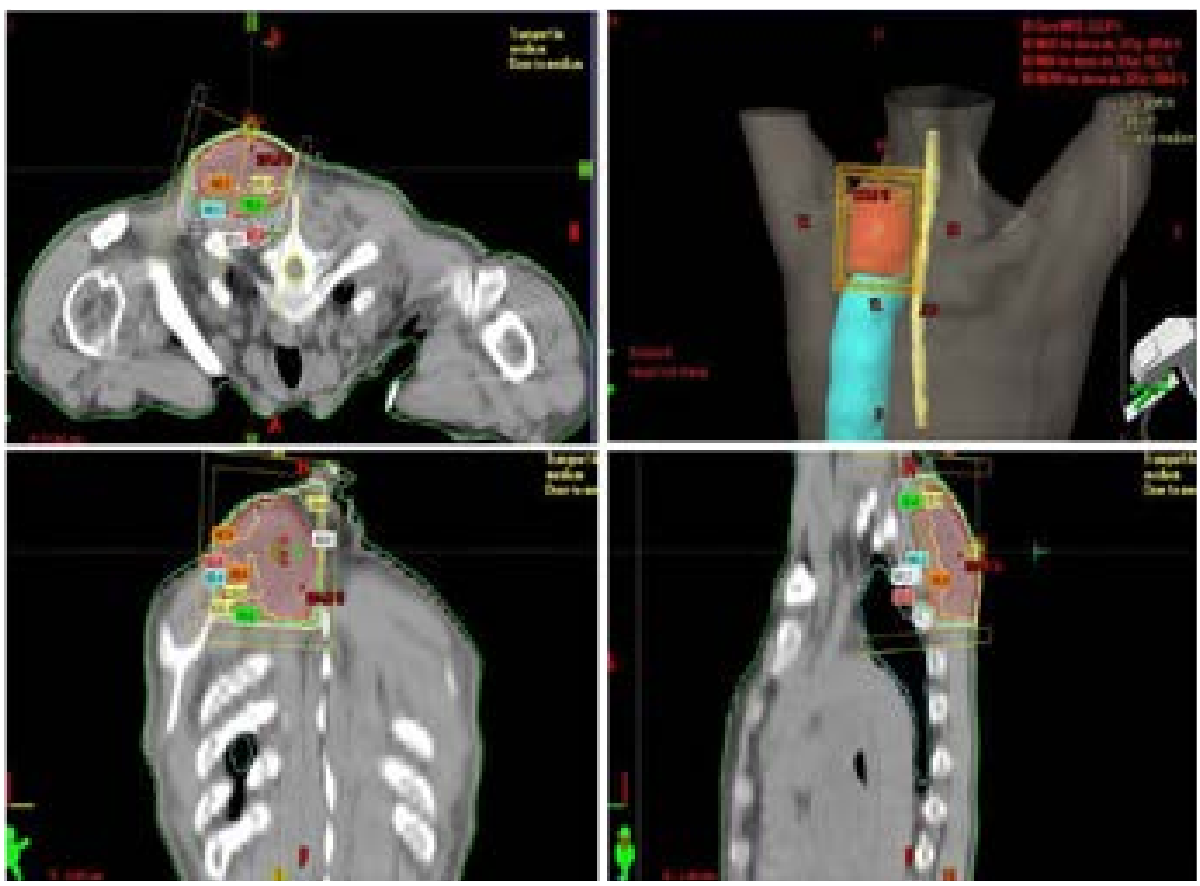

FIGURE 5: Treatment plan for left paravertebral lesion with isodose levels and contoured organs at risk (left lung in cyan and esophagus in yellow).

a total of 30 cycles of chemotherapy with trabectedin that have been well tolerated except for neutropenia G2 and mild anemia, improved with the use of growth factors. Also radiation treatments were well tolerated without late toxicity observed during follow up. After 41 months from metastatic disease and 28 months after the beginning of chemotherapy with trabectedin and complementary radiotherapy, the patient is in good general condition with good control of the disease. 

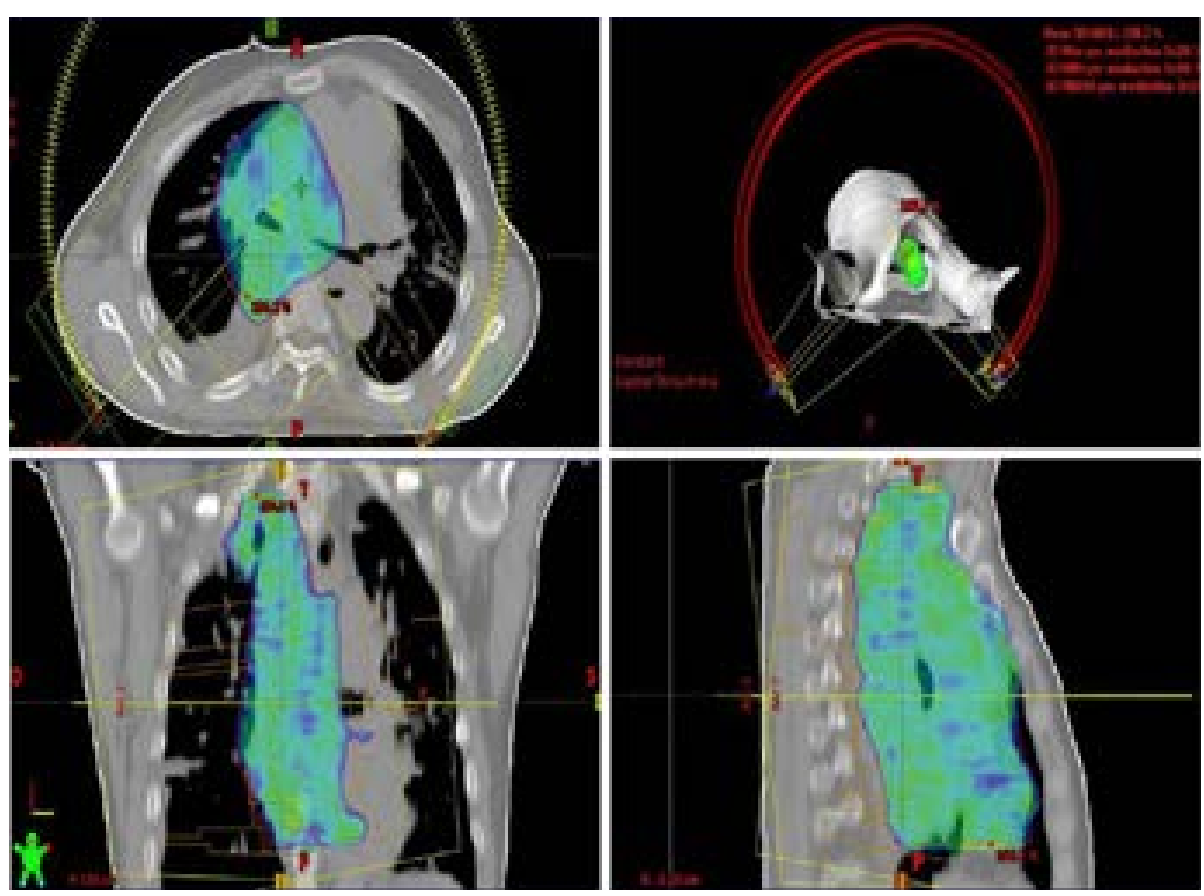

FIGURE 6: RapidArc plan with dose-distribution in color wash.

\section{Discussion}

EMC is classified as an intermediate grade malignancy due to its high rate of local recurrence and metastasis in a half of patients [13]. EMC commonly presents itself with enlarging soft tissue mass with non-specific symptoms as tightness, pain and the detection of a palpable mass [14].

The clinical evolution of EMC is difficult to predict. Nearly half of patients have one or more local recurrences within an average of 44 months [3]. In a review of 117 EMC cases MeisKindblom et al identified in the multivariate analysis that some clinical features, such as older patient age, larger tumour size greater than $10 \mathrm{~cm}$, and tumour location in the proximal extremity or limb girdle represent adverse prognostic factors [3]. Moreover, the incompleteness of the initial surgical resection, absence of surgical resection and discovery of metastases at diagnosis also appear to have a negative impact on survival [6]. Data regarding the histopathological features such as necrosis, mitotic activity, the degree of differentiation are discordant in the literature and do not appear influencing significantly the clinical outcomes [15].

Surgery is the principle treatment modality for EMC with the aggressive control of localized EMC as the primary management approach
$[3,8,12]$. Although the five-year survival rate is quite high of about $70-90 \%$, metastases are common (46-90\% of cases) $[3,6,12]$ and, despite seemingly promising local treatment, there is still a significant portion of EMC patients whose tumour is unresectable or recurrent or has already metastases to distant organs $[3,6]$.

Long-term prognosis is adverse with 30 $70 \%$ survival at ten years $[3,6]$. Survival after the onset of metastases varies widely due to different patient and disease characteristics. For these patients, therapeutic options are scarce and treatments are palliative only. Apart from some single cases reported in literature, no chemotherapeutic agent or agents combination have been demonstrated to achieve durable efficacy against EMC to date $[6,16]$. In the majority of patients the response to chemotherapy is unsatisfactory with a marginal efficacy to the standard first line doxorubicin containing regimen with a response rate of $15-$ $30 \%$ [17-18].

Drilon et al. reported the experience in 21 patients receiving 32 evaluable courses of chemotherapy with doxorubicin containing regimens comprising the largest subgroup and no significant radiologic or clinical responses were noted [8].

Some Authors reported a slight improvement of these limited benefits with the addition 
of ifosfamide, however such a combination regimen must be carefully weighed because of associated high toxicities. It is reasonable to employ this combination in patients with symptomatic, locally advanced or inoperable soft tissue sarcoma, since their combined effects may render such tumours resectable [18-19]. Recently, Ogura et al. reviewed their institutional series of 22 patients and no response in 4 cases treated with ifosfamide-based chemotherapy was reported [12].

Based on these data, it is apparent that long term benefit in the treatment of patients with unresectable or metastatic EMC is currently limited $[8,20]$.

The experience with targeted therapy in EMC is limited. Recently, Stacchiotti et al. reported a high activity of sunitinib in 10 patients with progressive metastatic translocated EMC with 6 patients having a partial response and 2 stable disease. Interestingly, all responsive patients expressed the typical EWSR1-NR4A3 fusion, while refractory cases carried the alternative TAF15-NR4A3 fusion [10].

The role of radiotherapy in EMC is not well-defined. Though sarcomas are tipically radioresistant tumors, there are reports in literature which suggest a high radiosensitivity of specific istologies, such as myxoid liposarcomas [21].

Recent cases demonstrate that in some cases radiotherapy affects the final prognosis of patients with locally advanced ECM. Conformal adjuvant radiotherapy at a dose of 60-66 Gray given on residual disease seems to induce significant symptomatic and radiological improvement [12,22-24]. Nevertheless, other Authors reported no benefits with adjuvant radiotherapy [25-26].

Recently, the effect of external beam radiotherapy (EBRT) on cancer-specific survival (CSS) for patients with localized EMC arising from soft connective tissues of the trunk and extremities have been reported. One hundred seventy-two patients were identified, diagnosed from 2004 to 2012. Ninety-four percent and $32 \%$ of 156 assessable patients underwent surgery and EBRT, respectively.

Authors observed a CSS of $97 \%$ vs. $85 \%$ and $94 \%$ vs. $85 \%$ in patients receiving EBRT vs. no EBRT, at 3 and 5 years, respectively, at median follow-up of 33 months ( $\mathrm{p}=0.01)$. Authors conclusions are that the receipt of EBRT was associated with a CSS benefit in localized EMC. Thus, aggressive local therapy, including EBRT, should be considered in these patients [27].

Radiotherapy in recurrent or metastatic disease has a palliative intent contributing to the local control of disease and amelioration of symptoms. Nevertheless, to date the information concerning the effect of radiotherapy on this disease is scarce. Some Authors reported a palliative benefit and sometimes tumor regression in patients underwent radiotherapy $[12,28]$.

Two cases of orbital mesenchymal chondrosarcoma in young adults presenting with proptosis and diminution of vision have been reported, which showed undifferentiated mesenchymal cells with islands of cartilage. Both patients underwent exenteration followed by chemotherapy and radiation therapy and were alive with healthy orbits after two years of follow-up [29]. Moreover, an almost complete disappearance of the right main bronchus obstruction following radiotherapy has been reported in an old woman with pulmonary metastasis from the EMC, and such a state had lasted for several years [30].

Intensity modulated radiotherapy (IMRT) seems to give advantages over conventional three-dimensional (3D) conformal treatment. In a patient with EMC of the lateral compartment of the leg in which the target volume was $50 \mathrm{~cm}$ in length and twisted around the surrounding bones. The IMRT plan produced a superior dose distribution to the patient as compared to the $3 \mathrm{D}$ conformal plan both in terms of dose conformity and homogeneity in the target volumes, and reduction of the maximum dose to the bone. The planning time of the IMRT plan was about 3-5 times shorter than that of the 3D conformal plan. It was demonstrated that the IMRT technique can be used not just for small tumors, but also for large and spiralshaped tumors close to critical organs. The IMRT method requires less planning time, and provides better target coverage with more sparing of critical structures. Thus, Authors concluded that when planning patients with multiple target volumes receiving different prescribed doses, the IMRT technique can more easily meet this requirement [31]. 
As a modern technique which combines dosimetric advantages of IMRT with shorter treatment delivery time [32], we chose Volumentric Modulated Arc Therapy (VMAT/ RapidArc) for the palliative treatment of mediastinal lymphadenopathies.

Trabectedin, a DNA-damaging agent with a mechanism of action that is different from that of traditional alkylating agents, is approved in Europe for the treatment of patients with advanced soft tissue sarcoma, after failure of anthracyclines and ifosfamide or who are unsuited to receive these agents. Trabectedin, at pharmacologically appropriated concentrations, has been reported to harbour a significant in vitro radiosensitising effect and induces cell cycle changes and apoptosis in several human cancer cell lines [33]. Trabectedin would sensitize sarcoma cells to radiation by synchronizing cells in the G2/M phase [34].

Clinical experiences with trabectedin and concomitant radiotherapy are very limited. A complete response of a recurrent-metastatic liposarcoma has been recently reported when trabectedin was used after radiotherapy on bone metastases [35]. An ongoing prospective study (TRASTS study: NCT02275286) is evaluating the synergic effect of trabectedin plus radiotherapy in soft tissue sarcomas, based on preclinical data [36].

In our patient we confirmed the synergistic effects of this combination. Trabectedin gave little benefit when utilized alone, but when radiotherapy was added, an impressive response was noted in all radio-treated sites providing evidence of a potent synergistic effect of this combination.

In conclusion, we judged that radiotherapy is highly effective in ECM, and when it is utilized in association with trabectedin, a potent synergistic effect could be obtained. 


\section{References}

Tsuneyoshi M, Enjoji M, Iwasaki H, Shinohara N. Extraskeletal myxoid chondrosarcoma--a clinicopathologic and electron microscopic study. Acta Pathol. Jpn. 31(3), 439-447 (1981).

Meis-Kindblom JM, Bergh P, Gunterberg B, Kindblom L-G. Extraskeletalmyxoid chondrosarcoma: a reappraisal of its morphologic spectrum and pronostic factors based on 117 cases. Am. J. Surg. Pathol. 23(6), 636650 (1999).

Smith MT, Farinacci CJ, Carpenter HA, Bannayan GA. Extraskeletalmyxoid chondrosarcoma: a clinicopathological study. Cancer. (1976).

Fletcher CDM, eds. World Health Organization (WHO) Classification of Tumours of Soft tissue and Bone. Pathology and Genetics. Lyon: IARC Press; (2013).

Antonescu CR, Argani P, Erlandson RA. et al. Skeletal and extraskeletalmyxoid chondrosarcoma: a comparative clinicopathologic, ultrastructural, and molecular study. Cancer. 3, 1504-1521 (1998).

McGrory JE, Rock MG, Nascimento AG, Oliveira AM. Extraskeletalmyxoid chondrosarcoma. Clin. Orthop. 382,185190 (2001).

Davis EJ, Wu YM, Robinson D, Schuetze SM, Baker LH, Athanikar J, Cao X, Kunju LP, Chinnaiyan AM, Chugh R. Next generation sequencing of extraskeletal myxoid chondrosarcoma. Oncotarget. 8(13), 21770-21777 (2017).

Drilon AD, Popat $S$, Bhuchar G, et al. Extraskeletal myxoid chondrosarcoma: a retrospective review from 2 referral centers emphasizing long-term outcomes with surgery and chemotherapy. Cancer. 113(12), 3364-3371 (2008).

Stacchiotti S, Dagrada GP, Morosi C, et al. Extraskeletal myxoid chondrosarcoma: tumor response to sunitinib. Clin. Sarc. Res. 2, 22 (2012).

Stacchiotti S. Activity of sunitinib in extraskeletalmyxoid chondrosarcoma. Eur. J. Cancer. 50(9), 1657-1664 (20140.

Kawaguchi S, Wada T, Nagoya $S$, et al. Multi-Institutional Study of 42 Cases in Japan. Extraskeletal myxoid chondrosarcoma: a Multi-Institutional Study of 42 Cases in Japan. Cancer.
97(5), 1285-1292 (2003).

Ogura K, Fujiwara T, Beppu Y. Extraskeletal myxoid chondrosarcoma: a review of 23 patients treated at a single referral center with long-term follow-up. Arch. Orthop. Trauma Surg. 132(10), 1379-1386 (2012).

Gupta SS, Khanna N, Jacobi A. Curious case of extraskeletal myxoid chondrosarcoma. Lung India. 34(2), 170-172 (2017).

Hisaoka M, Hashimoto $\mathrm{H}$. Extraskeletal myxoid chondrosarcoma: updated clinicopathological and molecular genetic characteristics. Pathol. Int. 55(8), 453-463 (2005).

Algros MP, Collonge-Rame MA, Bedgejian I. Différenciation neuroectodermique des chondrosarcomes extrasquelettiques myxoїdes: un classique? Ann. Pathol. 23(3), 244-248 (2003).

Han K, Sun YJ, Shen Z, et al. Extraskeletal myxoid chondrosarcoma: a case report of complete remission by chemotherapy and review of the literature. BMJ Case Rep. pii, bcr07.2009.2128 (2010).

Verma S, Chokkalingam P, McRae D. Chondrosarcoma of the greater cornu of the hyoid: a case report and literature review. Ear Nose Throat J. 87(10), E1-E5 (2008).

Stacchiotti. Anthracycline-based chemotherapy in extraskeletal myxoid chondrosarcoma: a retrospective study. Clin. Sarcoma. Res. 3, 16 (2013).

Pervaiz N, Colterjohn N, Farrokhyar $\mathrm{F}$, et al. A systematic meta-analysis of randomized controlled trials of adjuvant chemotherapy for localized resectable soft-tissue sarcoma. Cancer. 113(3), 573581 (2008).

Patel SR, Burgess MA, Papadopoulos NE, Linke KA, Benjamin RS. Extraskeletalmyxoid chondrosarcoma: long-term experience with chimiotherapy. Am. J. Clin. Oncol. 18(2), 161-163 (1995).

de Vreeze RS, de Jong D, Haas RL, Stewart F, van Coevorden F. Effectiveness of radiotherapy in myxoidsarcomasisassociated with a dense vascular pattern. Int. J Radiat. Oncol. Biol. Phys. 1, 72(5), 1480-1487.

Purkayastha A, Sharma N, Dutta V. Extraskeletal myxoid chondrosarcoma of nasopharynx: An Oncologic Entity Rarely Reported. Oman Med. J. 33(2), 159-162 (2018).

Oike N, Ogose A, Kawashima H, Umezu H, Inagawa S. Extraskeletal myxoid chondrosarcoma arising in the femoral vein: a case report. Skeletal. Radiol. 43(10), 1465-1469 (2014).

Bingaman $\mathrm{KD}$, Alleyne $\mathrm{CH} \mathrm{Jr}$, Olson JJ. Intracranial extraskeletal mesenchymal chondrosarcoma: case report. Neurosurgery. 46(1), 207-211 (2000).

Romañach MJ, Carlos R, Nuyens M, et al. Extraskeletalmyxoidchondrosarcoma of the masticator space in a pediatricpatient. J. Clin. Exp. Dent. 9(6), e825-e831 (2017).

Farhane FZ, Alami Z, Bouhafa T, Elmazghi A, Hassouni K. Paravertebral extraskeletal myxoid chondrosarcoma: a case report and review of the literature. Pan. Afr. Med. J. 21,2 13 (2015).

Kemmerer EJ, Gleeson E, Poli J, et al. Benefit of radiotherapy in extraskeletal myxoid chondrosarcoma: a propensity score weighted population-based analysis of the SEER database. Am. J. Clin. Oncol. 41(7), 674-680 (2018).

Jérôme-Marson V, Uro-Coste $\mathrm{E}$, Lacoste-Collin L, et al. Ann. Pathol. 23(3), 253-257 (2003).

Kaur A, Kishore P, Agrawal A, Gupta A. Mesenchymal chondrosarcoma of theorbit: a report of two cases and review of the literature. Orbit. 27(1), 63-67 (2008).

Mizushima Y, Fujita M, Hirata H, et al. Effective irradiation of a metastatic region of the lung in a patient with extraskeletal myxoid chondrosarcoma. Intern Med. 37(7), 625-627 (1998).

Chan MF, Chui CS, Schupak K, et al. The treatment of large extraskeletal chondrosarcoma of the leg: comparison of IMRT and conformal radiotherapy techniques. J. Appl. Clin. Med. Phys. 2(1), 3-8 (2001).

Taggar AS, Graham D, Kurien E, Grafe JL. Volumetric-modulated arctherapy versus intensity-modulated radiotherapy for large volume retroperitoneal sarcomas: A comparative analysis of dosimetric and treatment delivery parameters. J. Appl. Clin. Med. Phys. 19(1), 276-281 (2018). 
Romero J, Zapata I, Córdoba S, et al. In vitro radiosensitisation by trabectedin in human cancer cell lines. Eur. J. Cancer. 44(12), 1726-1733 (2008).

https://www.redjournal.org/article/ S0360-3016(17)33094-8/abstract
Kus T, Aktas G, Kalender $\mathrm{ME}$, et al. Complete response of a recurrent-metastatic liposarcoma with dedifferentiated histological features following the administration of trabectedin and review of literature. J. Cancer Res. Ther. 11(4), 974-976 (2015).
Gronchi A, Hindi N, Cruz J. Trabectedin and radiotherapy in soft tissue sarcoma (TRAST) study: An international, prospective, phaseI/II trial. A collaborative Spanish (GEIS) Italian (ISG), and French(FSG) groups study. J. Clin. Oncol. 35, (2017). 